\title{
Representaciones de naturaleza de la ciencia en libros de texto de química: indagando por los tópicos epistemológicos de Representación y Lenguajes ${ }^{1}$
}

\author{
Representations of Nature of Science in chemistry textbooks: \\ Inquiring for epistemological topics of Representation and \\ Languages
}

\author{
Representações de "natureza da ciência" em livros-texto de \\ Química: rastreando conteúdos sobre o tópico epistemológico \\ "Representação e Linguagens"
}

\author{
R.Y. Amador, N. Ospina y A. Adúriz
}

Recibido: abril 1 de 2018 - Aceptado junio 28 de 2018

\begin{abstract}
Resumen- El presente artículo ofrece a la comunidad académica que indaga en el campo de la Naturaleza de la Ciencia (NOS, siglas en inglés), algunas reflexiones y aportes teóricos que derivan de una tesis doctoral, con el objetivo de identificar la época epistemológica que orienta la NOS en dos libros de texto, específicamente en los aspectos de Representación y Lenguajes, para lo cual se construyeron redes sistémicas con el fin de analizar las afirmaciones textuales de los libros referentes
\end{abstract}

R. Y. Amador. Universidad de Norte, Instituto de Estudios en Educación -IESE, Barranquilla, Colombia, email: ryamador@uninorte. edu.co.

N. Ospina. Universidad de Buenos Aires, Buenos Aires, Argentina, email: nataliaospinaquintero@gmail.com.

Adúriz-Bravo, A. Universidad de Buenos Aires, Buenos Aires, Argentina, email: aadurizbravo@cefi ec.fcen.uba.ar

Como citar este artículo: Amador, R. Y., Ospina, N., Adúriz, A. Representaciones de naturaleza de la ciencia en libros de texto de química: Indagando por los tópicos epistemológicos de Representación y Lenguajes, Entre Ciencia e Ingniería, vol. 12, no. 24, pp. 116-123, julio-diciembre 2018. DOI: http://dx.doi.org/10.31908/19098367.3821

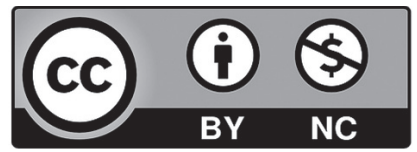

Attribution-NonCommercial 4.0 International (CC BY-NC 4.0) a los aspectos de Representación y Lenguajes. Los resultados muestran una ausencia de proposiciones referidas a los anteriores aspectos epistemológicos, y la omisión de proposiciones que se vinculan con algún tópico/época epistemológica confirma la falta de claridad respecto a una representación de NOS, resultados que ya han sido reportados en otras investigaciones. El aporte de esta investigación es la periodización epistemológica propuesta, considerándola un potente marco metacientífico para caracterizar representaciones de NOS con mayor finura conceptual.

Palabras clave - épocas epistemológicas, naturaleza de la ciencia, representación y lenguajes, tópicos epistemológicos.

Abstract - We are sharing some ideas and theoretical supports that comes from a doctoral thesis, with the scientific community that is inquiring about the nature of the science field (NOS). The goal of this work is to identify the epistemological time derives by NOS using two books, mainly in the representation and languages fields. Systematic networks were built in order to asses the textual statement derives from the books. no propositions related to Representation and Languages were found suggesting the absence or unclear thought about a NOS representation. These results have been already reported previously in other investigations. The highlight of this work is the proposal of the epistemology periodization, which is considered as a useful metascientific tool used for the characterization of NOS representation, with a better conceptual approach.

Keywords - epistemological periods, epistemological topic, nature of science, representation and languages. 
Resumo- Compartilhamos com a comunidade que investiga no campo da "Natureza da Ciência" ("NOS", a sigla em inglês), algumas reflexões e aportes teóricos que derivam de uma pesquisa de Doutorado. Neste artigo, propusemos a identificação da "fase epistemológica" que estaria norteando a "NOS" presente em dois livros didáticos - especificamente no que diz respeito aos tópicos epistemológicos da "Representação" e das "Linguagens". Construímos redes sistêmicas para a análise das afirmações textuais contidas nos livros, associadas aos referidos tópicos. E verificamos uma ausência de proposições relativas a eles. Essa espécie de omissão (isto é, de proposições vinculadas a alguma fase/tópico epistemológico) confirma a não clareza a respeito da representação de natureza da ciência fato já confirmado em pesquisas semelhantes. A contribuição deste estudo reside na periodização epistemológica proposta, a qual pensamos constituir um parâmetro metacientífico mais sofisticado e fértil para a caracterização das representações sobre NOS.

Palavras chave: Natureza da ciência; períodos epistemológicos; tópicos epistemológicos; representação e linguagens

\section{INTRODUCCIÓN}

E conceptualiza la naturaleza de la ciencia (NOS, por $\checkmark$ sus siglas en inglés) como un conjunto de contenidos metacientíficos seleccionados por su valor para la educación científica de la ciudadanía, sobre los cuales se han desarrollado diferentes perspectivas respecto de su organización y formas de efectuar la transposición didáctica a las clases [1]. Tales contenidos provienen de distintas escuelas y autores de la filosofía de la ciencia del siglo XX y, desde la perspectiva NOS en la que nos encuadramos, están ambientados en episodios paradigmáticos de la historia de la ciencia y advertidos por la sociología de la ciencia, contra el dogmatismo, el triunfalismo, el elitismo y el sesgo de género propios de las visiones de ciencia tradicionales, comúnmente calificadas de "cientificistas" o "tecnocráticas" [2] [3].

\section{REFERENTES TEÓRICOS}

A continuación se presentan algunos de los marcos teóricos y metodológicos que permitieron el desarrollo de la investigación.

\section{A. Concepción de NOS}

Afirma Adúriz-Bravo que la expresión NOS refiere al menos a tres objetos específicos, cada uno con su identidad propia y a la vez relacionados entre sí.

1. La NOS designa aquello que la ciencia es, o -más propiamente- las diversas conceptualizaciones que, desde las llamadas metaciencias (epistemología, historia de la ciencia, sociología de la ciencia, psicología de la ciencia, etc.), se han hecho a lo largo de la historia para responder a la pregunta “¿Qué es esa cosa llamada ciencia?”, que Alan Chalmers [4] extendió en el ámbito de la divulgación de la filosofía de la ciencia.

2. La NOS refiere también a un componente emergente dentro del currículo de ciencias naturales, aquel que se ocupa de reflexionar sobre el carácter de la actividad científica. La naturaleza de la ciencia, en tanto contenido a enseñar, es considerada hoy en día una parte fundamental, insoslayable y valiosa de la alfabetización científica.

3. Por último, la NOS evoca un área de investigación, innovación, docencia y extensión dentro de la didáctica de las ciencias naturales, que aborda los problemas surgidos de la necesidad de enseñar el conocimiento metacientífico, prescrito curricularmente, dentro del contexto de las clases de ciencias.

En el tercer sentido, la naturaleza de la ciencia, en tanto que construcción teórica, trabaja sobre los diversos y ricos aportes que las metaciencias (en especial, la epistemología y la historia de la ciencia) pueden hacer a la educación científica en todos sus aspectos: la formulación del currículo, la planificación de unidades didácticas, el diseño de materiales y textos, la formación inicial y continuada del profesorado de ciencias naturales y la solución de problemas específicos de enseñanza y aprendizaje [5].

\section{B. Las finalidades de la naturaleza de la ciencia}

Al discutir sobre las diversas funciones que una reflexión profunda sobre la ciencia (reflexión metateórica o de segundo orden) aporta a la formación de ciudadanos y ciudadanas autónomos, críticos, responsables, tolerantes y solidarios, y siguiendo las propuestas de diversos autores como Matthews [6], Driver et al. [7], McComas [8] y Adúriz-Bravo [5], es posible reconocer al menos tres finalidades fundamentales que puede desempeñar la NOS en la educación científica:

1. Una finalidad intrínseca: la NOS ha de ser una reflexión 'racional y razonable' [9] sobre las propias ciencias naturales, que sirva para analizarlas críticamente desde un segundo nivel de discurso. Por tanto, en la educación científica quedaría excluida para nosotros la presentación de formalismos abstractos per se, desconectados de su valor para pensar cuestiones interesantes y útiles alrededor de los dilemas que plantean actualmente la ciencia y la tecnología en la sociedad.

2. Una finalidad cultural: se puede trabajar la NOS desde distintas áreas curriculares (por ejemplo, las ciencias naturales junto con la filosofía, la historia, las ciencias sociales, la matemática y las artes), para destacar su valor histórico como creación intelectual humana, situando personajes e ideas en el contexto social amplio de cada época. Además, sabiendo NOS se puede generar una imagen de ciencia que se aleje de dos "ingenuidades" igualmente peligrosas: rechazarla frontalmente como una superchería o mistificación opresora que da origen a todos los males de la humanidad (posición relativista extrema), o admirarla acríticamente como un conjunto de verdades "dogmáticas" impuestas por la tecnocracia (posición positivista extrema).

3. Una finalidad instrumental: la NOS ha mostrado ser una herramienta valiosa para mejorar la enseñanza y el aprendizaje de los contenidos científicos. Las metaciencias son fuente de advertencias y consejos para identificar y atacar los obstáculos didácticos más importantes que aparecen asociados a la enseñanza de los grandes modelos 
de la historia de la ciencia, tales como la mecánica clásica, el cambio químico, la genética, la tectónica de placas o la teoría de la evolución. Un sólido conocimiento metacientífico permitiría que los y las estudiantes vinculen mejor los contenidos y formas de pensar de las ciencias naturales con el conocimiento del sentido común que circula en la sociedad. Y, por otra parte, la reflexión generada desde la epistemología y las demás metaciencias proveerían de herramientas, materiales y enfoques útiles para la tarea cotidiana de enseñar ciencias naturales en las aulas [6].

\section{Tópicos epistemológicos para caracterizar concepciones de NOS}

Los Tópicos epistemológicos son ideas estructurantes que permiten organizar contenidos de la filosofía de la ciencia o epistemología con el propósito de analizar y evaluar la actividad científica desde distintas perspectivas de esta metaciencia. Son ideas transpuestas que para nosotros tienen una vinculación sustancial con la didáctica de las ciencias naturales [10] [11].

Por ejemplo, el tópico "Representación" describe la forma en que los científicos representan o modelizan los fenómenos y "Lenguajes" refiere a la forma en que los científicos acuden a recursos de tipo simbólico, semiótico, expresivo, para definir, describir, expresar y comunicar las teorías o modelos científicos.

\section{Periodización epistemológica para caracterizar concepciones de NOS}

La epistemología como saber de segundo orden o metaciencia, es una disciplina muy activa y su producción se ha organizado en varias épocas y escuelas de investigación, las cuales han discutido entre ellas en pos de hacer prevalecer ciertas visiones sobre otras [12]. En esa extensa y heterogénea producción se deben seleccionar determinados aspectos y perspectivas en función de las intencionalidades generales de la educación científica y de la didáctica de las ciencias naturales [13].

La periodización propuesta comprende cinco épocas epistemológicas [10], y en ellas los tópicos epistemológicos adquieren significado específico:

\section{El Positivismo Lógico y la Concepción Heredada}

A menudo se sitúa el inicio de la epistemología institucionalizada en la constitución del Círculo de Viena en la década del 20 del siglo pasado. En el positivismo lógico se puede reconocer una primera formalización de la imagen popular o folk de la ciencia [14]; los epistemólogos que trabajan bajo estos presupuestos teóricos enfatizan en el aspecto metodológico de la actividad científica, privilegiando un enfoque investigativo "sintáctico" (es decir, lógico-lingüístico) con la intención de generar un análisis riguroso de la estructura y validez del conocimiento científico.

En esta época se destaca el análisis sincrónico de las teorías, es decir, el análisis de la estructura de una teoría científica separada de su evolución en el tiempo. Otro aspecto es que se concibe a la ciencia como un conjunto de proposiciones, las cuales dicen cómo es el mundo. También es característica de esta época la denominada unificación de la ciencia, la cual debe llevarse a cabo reduciendo todas las proposiciones científicas a proposiciones observacionales y estas, a su vez, expresarlas en un lenguaje primario, original y objetivo.

\section{El Racionalismo Crítico}

En este momento epistemológico, un aspecto central es proponer que el progreso científico se produciría a partir de la falsación de las teorías y su reemplazo temporal por otras más satisfactorias, a través de sucesivas "conjeturas y refutaciones" [15] [16]. Una teoría científica se prefiere si logra explicar más hechos, si es más englobante. Por ejemplo, es preferible para un científico la física de Newton que las leyes de Kepler, ya que la física de Newton explica el movimiento de los planetas, pero de manera más amplia. Aunque es claro que una teoría con mayor poder explicativo es necesariamente menos probable que una que tenga menor poder de explicación. Así, al elegir las más englobante, el científico escoge la menos probable.

Otra de las características de este periodo es la de aceptar prácticamente cualquier hipótesis científica sin evaluación previa, pero una vez aceptada se hará todo lo posible por refutarla, situación que no sucede en la época anterior. Otro aspecto importante es que, por ejemplo, cuando un científico sugiere una teoría ha de someterla a un proceso de evaluación ante la comunidad científica; los representantes de esta, en principio, intentarán refutarla, y si lo logran será un triunfo para la ciencia, ya que se sabrá con seguridad que esa teoría no era verdadera, situación que permite acercarse más a la verdad, dado que se ha eliminado un error en la ciencia [17].

\section{La Nueva Filosofía de la Ciencia}

Los representantes de esta fase epistemológica (el propio Kuhn, junto con Imre Lakatos y otros) se ocupan de los hechos sociales de la ciencia, enfatizando en constructos tales como los de comunidad científica, progreso/cambio o contexto social y cultural. La nueva filosofía de la ciencia, al menos en esta versión destacada, genera su propuesta teórica con el apoyo de la historia de la ciencia, fuente que provee los insumos empíricos con los cuales se puede interpretar la "dinámica" científica.

Se podría decir que esta época se desarrolla en la década del 60 del siglo pasado; sus representantes se caracterizan por tener dos grandes intereses: estudiar la historia de la ciencia y confrontar las distintas tesis de la concepción heredada. Como resultado de esta óptica epistemológica diferente, surgieron propuestas metodológicas alternativas a la época anterior. Por ejemplo, se asumen las teorías no como sistemas axiomáticos acabados, sino como "organismos" más o menos complejos que se desarrollan en el tiempo, formulando categorías tales como paradigmas, proyectos de investigación científica, etc., siendo estos las unidades de análisis, comprensión, explicación y representación del conocimiento científico.

Una de sus características es la de no considerar a la ciencia como una simple estructura lógica sino como un constructo 
social, es decir, una actividad más de una comunidad -la científica-, resaltando el contexto de descubrimiento como ámbito importante en la comprensión del conocimiento científico.

\section{El Postkuhnianismo}

Es una etapa epistemológica que se caracteriza por las críticas a los presupuestos del racionalismo y del relativismo "puros". Por ejemplo, Feyerabend no desarrolla un modelo de progreso científico, dado que considera que la ciencia no es un sistema "unificado", del cual se puedan expresar afirmaciones en general [17]. Los representantes de esta época desarrollan la tesis de que tanto la metodología inductivista del neopositivismo, como la metodología deductivista del racionalismo crítico, son "limitantes" para los científicos.

Por tanto, los representantes de esta posición señalan que la pretendida neutralidad y objetividad en la ciencia no se logra, por ejemplo, concuerdan en que los términos teóricos están cargados de teoría, tal como se venía sustentando en épocas epistemológicas anteriores, y en que toda observación depende de la teoría con que se observe el mundo; por tal razón, se sustenta que no son los términos observacionales los que dan sentido a los teóricos, sino los teóricos los que determinan a los observacionales.

Otro de los aspectos que caracteriza este momento, es que ninguna de las metodologías propuestas a lo largo de la historia han tenido éxito (ni el inductivismo ingenuo, ni el probabilista; ni el falsacionismo ingenuo ni sofisticado), cualquier metodología es incompatible con la historia de la ciencia, pues esta es mucho más rica de lo que permite cualquier método, motivo por el que se afirma que no existe un único método en la ciencia; por ejemplo, Feyerabend [17] plantea que cada teoría determina su propio método y, por ende, su propia racionalidad científica.

\section{Visiones contemporáneas}

Esta última época está conformada por una pluralidad de escuelas epistemológicas que conviven con diferente grado de armonía entre ellas, y distinto grado de tolerancia de las unas con las otras. Para los fines de este trabajo, se toma solo una de las propuestas actualmente vigentes. Se podría afirmar sobre una se esas propuestas, sin ser demasiado arriesgados, que la: Concepción semántica de las teorías cientificas tiene alta acogida en la comunidad de epistemólogos y suscita la atención de didactas de las ciencias. Esta época epistemológica, sobre todo en sus últimas tres décadas, se caracteriza justamente por las críticas que se realizan a los presupuestos del racionalismo y del relativismo "puros".

Es decir, actualmente se impugna tanto la creencia de que solo serán científicas aquellas teorías que puedan ser valoradas en términos universales y que sobrevivan a la prueba de la experimentación, como la creencia de que las teorías se juzgan a partir de los sistemas de valores de los individuos o comunidades que las sostienen [18].

Los epistemólogos que trabajan bajo la concepción semántica se concentran en el significado y uso de las teorías científicas y no tanto en su forma o estructura. Los planteamientos generados por el semanticismo apuntan a que la relación entre los fenómenos ("realidad") y lo que decimos sobre ellos ("representación"), aparece mediada por los modelos científicos en tanto son representaciones abstractas del mundo [19]. En esta perspectiva, tres autores formulan propuestas teóricas vinculadas a la categoría de modelo científico: Fred Suppe, Bas van Fraassen y Ronald Giere [19].

Las ideas de estos tres epistemólogos apuntan a que no existe una relación tan directa entre aquello que decimos y los fenómenos en sí, sino que esa relación está mediada por los modelos en tanto que representaciones abstractas del mundo, representaciones que no son reducibles completamente ni a enunciados ni a realidad [19].

Algunas coincidencias teóricas que se pueden identificar en entre los planteamientos de Suppe, Giere y van Fraassen, son [19]:

- Una "reinterpretación" de las teorías científicas como conjuntos o clases de modelos, lo que proporciona una imagen más satisfactoria de la relación entre las teorías científicas y el mundo real, a causa del fuerte papel de mediador cumplido por los modelos.

- La consideración de las teorías como algo más que "tinglados" formales de enunciados teóricos deductivamente engarzados, con la inclusión fuerte de los hechos intencionadamente interpretados por esos enunciados.

Así, una teoría es-almenos en parte-una familia demodelos, los cuales presentan relaciones lógicas y experimentales que aseguran cierta coherencia al conjunto. Esta familia puede ser descrita de varios modos, mediante enunciados diferentes, en lenguajes diversos, y ninguna formulación lingüística tiene ningún estatuto privilegiado. Específicamente, no se atribuye mayor importancia a la axiomatización como tal, e incluso la teoría puede no ser axiomatizable en sentido no trivial [20]. Se puede entonces, siguiendo a Pablo Lorenzano [21], afirmar que los autores semanticistas comparten el espíritu formalista del período clásico, aunque no la "letra" de las herramientas abstractas utilizadas para cumplir sus objetivos.

Algunos investigadores en didáctica acuden a los planteamientos de Giere [22] porque les encuentran potencia teórica para iluminar la construcción del conocimiento científico escolar [23] [24] [25]. Giere pone en el foco de su meta-análisis de la ciencia su naturaleza profundamente cognitiva: lo que se busca es la generación o construcción de conocimiento que dé sentido y sirva para intervenir, y propone que un "modelo teórico" es el medio representacional privilegiado con el que los científicos capturan el mundo tanto para sí mismos como para los demás; considera también que en la construcción y reconstrucción de modelos científicos juegan un papel importante las hipótesis teóricas, que, a diferencia de los modelos, sí son entidades lingüísticas. Ellas afirman un cierto tipo y grado de relación entre el modelo y un sistema real dado, y de ellas cabe predicar verdad y falsedad.

Adúriz-Bravo e Izquierdo [19] acuden a los planteamientos 
de Giere, recuperando la idea de que la categoría de modelo teórico se relaciona sustantivamente con dos elementos:

- el conjunto -amplio y heterogéneo- de recursos simbólicos (expresivos) que se utiliza para definirlo; y

- el sistema real al cual viene a modelizar, con el cual mantiene la relación de parecido antes expuesta, que técnicamente se llama "similaridad".

Entonces, según esta analogía, en la ciencia escolar las teorías también estarían compuestas de varios elementos: una población de modelos ligados entre sí, diversos sistemas semióticos para describirlos con profundidad, y varias hipótesis conectando esos modelos con sistemas en el mundo real.

En la transposición del semanticismo de Giere a la didáctica de las ciencias, se propondría que una teoría determina una clase de modelos científicos escolares para algo, para dar cuenta de ciertos datos, fenómenos o experiencias correspondientes a determinado ámbito de la realidad sobre el cual el estudiantado se pregunta y tiene interés en intervenir. Buena parte de la identificación de una teoría en la ciencia escolar consistiría entonces en la identificación de esos fenómenos empíricos, socialmente relevantes, de los que pretende predicar.

\section{E. Afirmaciones con alta carga teórica que refieren a los tópicos de Representación y Lenguajes}

En la Tabla I y II se presentan las afirmaciones con alta carga teórica referentes a los tópicos epistemológicos de Representación y Lenguajes. Estas afirmaciones se entienden como enunciados semánticamente potentes que describen o se refieren a tópicos epistemológicos. Cada tópico epistemológico representa algún aspecto de la actividad científica, lo que permite caracterizar concepciones de NOS en cualquier muestra de estudio [10] [11] [26]. Entiéndase PL/CH (Positivismo lógico/ Concepción Heredada); RC (Racionalismo crítico); NFC (Nueva filosofía de la ciencia); PK (Postkuhnianismo) y VC (Visiones contemporáneas). Estas afirmaciones surgen del análisis y selección intencionada de la lectura de tres libros especializados en epistemología; las preguntas que permitieron la selección de las afirmaciones que aluden a estos dos tópicos epistemológicos son: ¿Cuáles son los modos de representación a los cuales acuden los científicos para dar cuenta del mundo? Y, ¿Cuáles son los lenguajes científicos a los que acuden los científicos para representar el mundo? [cita de mi tesis]; la validación se hizo con expertos, tanto del campo de la filosofía de la ciencia como de la didáctica de las ciencias.

\section{METODOLOGÍA}

La combinación de enfoques cualitativos y cuantitativos es una tendencia actual en la investigación en didáctica de las ciencias naturales, situación que se observa, por ejemplo, en el número creciente de tesis doctorales en el campo. La investigación llevada a cabo se suma a tal tendencia, aunque se inscribe básicamente en un enfoque cualitativo específicamente en lo referido a la generación de las categorías
TABLA I

AFIRMACIONES CON ALTA CARGA TEÓRICA REFERENTES AL TÓPICO EPISTEMOLÓGICO REPRESENTACIÓN

\begin{tabular}{|c|c|c|}
\hline \multicolumn{3}{|r|}{ ACTs } \\
\hline \multirow{5}{*}{ 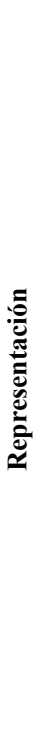 } & $\mathrm{PL} / \mathrm{CH}$ & $\begin{array}{l}\text { Las teorías científicas están conformadas por un con- } \\
\text { junto de afirmaciones lógicas que pretenden, una vez } \\
\text { interpretadas, decir algo del mundo. }\end{array}$ \\
\hline & $\mathrm{RC}$ & $\begin{array}{l}\text { Las teorías científicas son conjeturas arriesgadas que } \\
\text { se aceptan provisionalmente en tanto no son refuta- } \\
\text { das. }\end{array}$ \\
\hline & NFC & $\begin{array}{l}\text { Las teorías científicas son entidades estructural- } \\
\text { mente complejas formadas por principios generales } \\
\text { ubicados en un núcleo y con un conjunto de supues- } \\
\text { tos generales que se ponen a prueba empíricamente, } \\
\text { localizados en un cinturón. }\end{array}$ \\
\hline & PK & $\begin{array}{l}\text { La teoría científica es un conjunto relativamente es- } \\
\text { pecífico de doctrinas, leyes, hipótesis o principios } \\
\text { relacionados, que se usan para hacer predicciones } \\
\text { experimentales u observacionales y ofrecer explica- } \\
\text { ciones de fenómenos naturales. }\end{array}$ \\
\hline & $\mathrm{VC}$ & $\begin{array}{l}\text { Presentar una teoría no es presentar una clase de ax- } \\
\text { iomas, las teorías no se identifican metateóricamente } \\
\text { con conjuntos de enunciados; presentar una teoría es } \\
\text { presentar una clase de modelos, las teorías se identi- } \\
\text { fican metateóricamente como conjuntos de modelos. }\end{array}$ \\
\hline
\end{tabular}

TABLA II

AFIRMACIONES CON ALTA CARGA TEÓRICA REFERENTES AL TÓPICO EPISTEMOLÓGICO LENGUAJES

\begin{tabular}{|c|c|c|}
\hline \multicolumn{3}{|r|}{ ACTs } \\
\hline \multirow{5}{*}{ 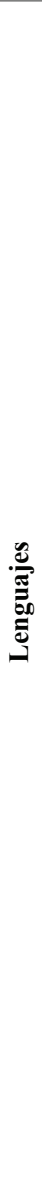 } & $\mathrm{PL} / \mathrm{CH}$ & $\begin{array}{l}\text { El vocabulario formal de una teoría es el vocabu- } \\
\text { lario de apoyo que proporciona el lenguaje o instru- } \\
\text { mental formal, y que en algunos casos puede incluir } \\
\text { partes muy elevadas de la matemática; el vocabu- } \\
\text { lario observacional refiere a entidades directamente } \\
\text { observables y a propiedades y relaciones entre ellas } \\
\text { directamente observables, y el vocabulario teórico } \\
\text { refiere a entidades, propiedades y relaciones no di- } \\
\text { rectamente observables postuladas para dar cuenta } \\
\text { de los fenómenos. }\end{array}$ \\
\hline & $\mathrm{RC}$ & $\begin{array}{l}\text { Si las conclusiones deducidas de la teoría sometida } \\
\text { a contrastación entran en conflicto con los enuncia- } \\
\text { dos básicos admitidos por la comunidad científica, } \\
\text { entonces la teoría debe ser considerada errónea y, } \\
\text { por tanto, queda refutada y debe ser sustituida por } \\
\text { otra mejor. Si, por el contrario, estas conclusiones } \\
\text { no entran en conflicto con ningún enunciado básico } \\
\text { admitido, la teoría ha pasado con éxito la prueba } \\
\text { por esta vez. }\end{array}$ \\
\hline & $\mathrm{NFC}$ & $\begin{array}{l}\text { Las leyes científicas son generalizaciones simbóli- } \\
\text { cas que funcionan como expresiones que se dan } \\
\text { dentro de un sistema matemático puro. }\end{array}$ \\
\hline & PK & $\begin{array}{l}\text { Una teoría T ha resuelto un problema empírico, si T } \\
\text { funciona (significativamente) en cualquier esquema } \\
\text { de inferencia cuya conclusión es un enunciado del } \\
\text { problema. Un problema empírico queda resuelto } \\
\text { por una teoría si de esta, junto con determinadas } \\
\text { condiciones iniciales, se puede derivar un enuncia- } \\
\text { do, habitualmente solo aproximado, del problema. }\end{array}$ \\
\hline & VC & $\begin{array}{l}\text { Presentar una teoría es presentar una familia de } \\
\text { modelos. Esta familia puede ser descrita de vari- } \\
\text { os modos, mediante enunciados diferentes en len- } \\
\text { guajes diferentes, y ninguna formulación lingüística } \\
\text { tiene estatuto privilegiado sobre las demás. }\end{array}$ \\
\hline
\end{tabular}


y a la obtención, análisis y validación de los datos-. En este sentido, los resultados obtenidos no conducen a afirmar que podrían ser exactamente replicados en el análisis de otros libros de texto, pero el instrumento de análisis que se diseñó puede ser utilizado para caracterizar concepciones o visiones de NOS, tanto en libros como en otras muestras de estudio (profesores, estudiantes, científicos, etc.).

En esta investigación se construyeron redes sistémicas, las cuales se proponen como una metodología para ordenar datos cualitativos [27] provenientes de instrumentos tales como cuestionarios abiertos, observaciones de aula, entrevistas, etc.; se plantea que detrás de cada afirmación o palabra oral o escrita en el contexto de una idea, hay un significado que no se expresa directamente con las palabras generadas.

Una red sistémica permite conectar las diferentes descripciones de los datos con las posibles interpretaciones, y establecer relaciones entre los significados, permitiendo estructurar los datos recolectados de acuerdo con una categorización previamente establecida por los investigadores. En este estudio, las categorías son las afirmaciones con alta carga teórica de los tópicos de Representación y Lenguajes. La forma de organizar la red se deriva de una selección que realiza el investigador en función de esos objetivos; esa forma involucra comúnmente la introducción de términos que facilitan y orientan la lectura de los datos.

\section{A. Elementos pragmáticos de la investigación}

Se analizaron en total 2 (dos) libros de texto de química, colombianos, diseñados para el nivel secundario, vigentes entre 2008 y 2015. La elección de los libros se realizó bajo el criterio que fueran los de mayor venta en el mercado, al no haber recomendación oficial desde el Ministerio de Educación (Tabla III).

En los dos libros mencionados se realizó la lectura de cada capítulo referido (o en los que se hacen menciones que pueden ser vinculadas a NOS); en esta primera lectura se seleccionaron 24 afirmaciones, las cuales poseen alguna relación semántica con la NOS. Un segundo momento fue el de establecer correlaciones semánticas entre las afirmaciones seleccionadas y los tópicos Representación o Lenguajes para cada época epistemológica.

TABLA III

LIBROS DE ENSEÑANZA DE LA QUÍMICA PARA EL NIVEL SECUNDARIO COLOMBIANO

\begin{tabular}{cccc}
\hline $\begin{array}{c}\text { Nombre del } \\
\text { libro }\end{array}$ & $\begin{array}{c}\text { Año de } \\
\text { Publicación }\end{array}$ & Editorial & Autores \\
\hline Química@1 & 2007 & Norma & $\begin{array}{c}\text { Castelblanco, Y. B. y } \\
\text { Sánchez de Escobar, } \\
\text { M. }\end{array}$ \\
\hline $\begin{array}{c}\text { Química } \\
\text { Inorgánica }\end{array}$ & & & $\begin{array}{c}\text { Mondragón, C. H., } \\
\text { Peña, L. Y., Sánchez } \\
\text { de Escobar, M. y } \\
\text { Arbeláez, F. }\end{array}$
\end{tabular}

\section{B. Interpretación de los datos}

Una vez desarrollado el proceso de correlación descrito anteriormente y recurriendo a la construcción de las redes sistémicas, se estableció que, de las veinticuatro proposiciones determinadas inicialmente de los dos libros de texto, tan solo una afirmación se correlaciona con el tópico de Representación y ninguna con el tópico Lenguajes; las otras veintitrés afirmaciones se vinculan con otros tópicos epistemológicos referentes a la NOS.

En la tabla IV se muestra la correlación entre la teoría y el dato, es decir, entre la afirmación con alta carga teórica del tópico Representación relacionada con la época epistemológica Racionalismo Crítico, y la afirmación que aparece en uno de los libros analizados. La correlación se argumenta ya que ambas afirmaciones se refieren al mismo tópico/época epistemológica, pero la distinción entre cada una de ellas es la densidad teórica de cada proposición. Para sostener la idea de que ambas afirmaciones (teoría-dato) metadiscursean acerca del mismo aspecto de la actividad científica (Representación), se propusieron afirmaciones puente, referentes al mismo tópico/época, pero con menor densidad teórica.

TABLA IV

\begin{tabular}{|c|c|c|c|}
\hline \multicolumn{4}{|c|}{ AFIRMACIONES CON SENTIDO SEMÁNTICO } \\
\hline $\begin{array}{c}\text { Afirmación } \\
\text { con alta carga } \\
\text { teórica } \\
\begin{array}{c}\text { Representa- } \\
\text { ción }\end{array}\end{array}$ & $\begin{array}{l}\text { Afirmación } \\
\text { puente }\end{array}$ & $\begin{array}{c}\text { Afirmación } \\
\text { puente }\end{array}$ & $\begin{array}{c}\text { Dato } \\
\text { Afirmación } \\
\text { textual }\end{array}$ \\
\hline $\begin{array}{c}\text { Las teorías } \\
\text { científicas son } \\
\text { conjeturas } \\
\text { arriesgadas } \\
\text { que se aceptan } \\
\text { provisional- } \\
\text { mente en tanto } \\
\text { no son refuta- } \\
\text { das. }\end{array}$ & $\begin{array}{c}\text { Las afirma- } \\
\text { ciones que } \\
\text { conforman } \\
\text { las teorías son } \\
\text { susceptibles } \\
\text { de refutación, } \\
\text { es decir, que } \\
\text { es posible que } \\
\text { una o varias } \\
\text { observaciones } \\
\text { contradigan la } \\
\text { teoría. }\end{array}$ & $\begin{array}{l}\text { Las teorías se } \\
\text { constituyen } \\
\text { de afirma- } \\
\text { ciones que } \\
\text { pretenden } \\
\text { explicar el } \\
\text { mundo y se } \\
\text { refutan o } \\
\text { corroboran } \\
\text { a partir de } \\
\text { resultados } \\
\text { empíricos. }\end{array}$ & $\begin{array}{c}\text { Una teoría está } \\
\text { constituida por } \\
\text { una serie de } \\
\text { hipótesis que } \\
\text { conforman un } \\
\text { sistema deducti- } \\
\text { vo, no explicativa } \\
\text { y se aplica a un } \\
\text { conjunto bien } \\
\text { definido de fenó- } \\
\text { menos, por lo que } \\
\text { no puede tomarse } \\
\text { como una verdad } \\
\text { absoluta. }\end{array}$ \\
\hline
\end{tabular}

El escaso número de afirmaciones referentes al tópico epistemológico Representaciones, fomentan cuestionamientos como: ¿Por qué el tópico Representaciones no es para los autores y editores de libros de texto un aspecto relevante a resaltar? ¿Qué concepción de Representación de la actividad científica se oculta ante esta aparente ausencia?; enseñar química como ciencia escolar, ¿consiste solo en enseñar teorías o modelos científicos escolares sin resaltar las distintas representaciones que hacen de la química una ciencia, y ciencia escolar?

La ausencia de afirmaciones que refieran al tópico Lenguajes promueve los siguientes interrogantes: ¿Por qué el tópico Lenguajes no es para los libros de texto un aspecto relevante a destacar? ¿Qué concepción del lenguaje 
científico subyace a tal exclusión?; enseñar química como ciencia escolar, ¿consiste solo en enseñar teorías o modelos científicos escolares sin resaltar las distintas mediaciones lingüísticas en la construcción de la química?

\section{Conclusiones}

La aparente ausencia de proposiciones referidas a los tópicos epistemológicos de Representación y Lenguajes concuerdan con lo ya evidenciado por Abd-el-Khalick, Waters \& Le [29], y es que algunos libros de texto de ciencias naturales no presentan una visión clara de NOS; la omisión de proposiciones que se vincule con algún tópico/época epistemológica confirma la falta de claridad con respecto a una representación de NOS.

La periodización epistemológica propuesta es un adecuado marco metacientífico para caracterizar representaciones de NOS con mayor finura conceptual. Tal periodización recupera contenidos de la epistemología, los cuales no han sido asumidos explícitamente en las distintas investigaciones que indagan por las visiones de NOS en los libros de texto de ciencias naturales; tal especificidad permite afirmar que los libros de textos analizados carecen de una visión clara con respecto a esos tópicos epistemológicos y de la naturaleza de la ciencia.

\section{REFERENCIAS}

[1] Vázquez, Á. A. y Manassero, M. A. "Una taxonomía para facilitar la enseñanza explícita de la naturaleza de la ciencia y su integración en el desarrollo del currículo de ciencias". Interacções. vol. 11, no. 34, 2016.

[2] Adúriz-Bravo, A., “¿Qué naturaleza de la ciencia hemos de saber los profesores de ciencias? Una cuestión actual de la investigación didáctica". Tecne, Episteme y Didaxis, pp. 23-33, 2005.

[3] Amador-Rodríguez, R. Y. and A. Adúriz-Bravo, A., "Consensus and Dissent Around the Concept of Nature of Science in the IberoAmerican Community of Didactics of Science". Teaching Science with Context. Springer, Cham, 31-47, 2018.

[4] Chalmers, A. F., ¿Qué es esa cosa llamada ciencia? Madrid, Siglo XXI 1984.

[5] Adúriz-Bravo, A., "Áreas de investigación en la didáctica de las ciencias experimentales: La naturaleza de la ciencia”. En: C. Merino Rubilar, A. Gómez Galindo, A. Adúriz-Bravo (coords.): Áreas y estrategias de investigación en la didáctica de las ciencias experimentales, pp. 111-125. Bellaterra: Servei de Publicaciones de la UAB. 2008.

[6] Matthews, M. R., "Un lugar para la historia y la filosofía en la enseñanza de las ciencias". Comunicación, lenguaje y educación, vol. 3, no. 11-12, pp. 141-156, 1991

[7] Driver, R., Leach, J., Millar, R. y Scott, P., Young people's images of science. Buckingham, UK: Open University Press, 1996.

[8] McComas, W. F., (Ed.). The Nature of Science in Science Educations. Rationales and Strategies. Kluwer Academic Publishers, 1998.

[9] Izquierdo, M. y Aliberas, J., Pensar, actuar i parlar a la classe de ciències. Per un ensenyament de les ciències racional i raonable. Bellaterra: Servei de Publicacions de la UAB. 2004.

[10] Amador-Rodríguez, R. Y., La Naturaleza de la Ciencia Representada en Libros de Texto de Química Latinoamericanos. Tesis doctoral. Universidad Nacional del Comahue, Neuquen - Argentina, 2018.

[11] Amador-Rodríguez, R. Y. y Adúriz-Bravo, A., "Afirmaciones epistemológicas con "alta carga teórica" que pueden tener incidencia en la didáctica de las ciencias: Un estudio comparativo". Ciência \& Educação, vol. 20, no. 2, pp. 433-447, 2014. DOI: http://dx.doi. org/10.1590/1516-73132014000200011

[12] Herrón, M. A., "Epistemology and epistemic cognition: The problematic virtue of relativism and its implications for science education”. Zona Próxima, no. 12, pp. 96-107, 2010.

[13] Adúriz-Bravo, A., Izquierdo i Aymerich, M. y Estany, A., "Una propuesta para estructurar la enseñanza de la Filosofía de la Ciencia para el profesorado de Ciencias en formación". Enseñanza de las Ciencias, vol. 20, no. 3, pp. 465-476, 2002.

[14] Adúriz-Bravo, A., Integración de la epistemología en la formación del profesorado de ciencias. Tesis Doctoral. Bellaterra: Universitat Autònoma de Barcelona, 2001.

[15] Díez, J. A. y Moulines, U., Fundamentos de filosofía de la ciencia. Barcelona: Ariel, $2^{a}$ ed., 1999.

[16] Mellado, V., "Cambio didáctico del profesorado de ciencias experimentales y epistemología”. Enseñanza de las Ciencias, vol. 21, no. 3, pp. 343-358, 2003.

[17] Diéguez, A., Filosofia de la ciencia. España: Editorial Biblioteca Nueva, 2005

[18] Armero, J., Naturalización y relativismo epistemológicos. Series Filosóficas. Madrid, UNED, 1999.

[19] Adúriz-Bravo, A., y Izquierdo-Aymerich, M., "Towards model based science education research". Revista de Educación en Ciencias/ Journal f Science Education, vol.10, no. Especial, p. 202, 2009.

[20] Díez Calzada, J. A., "La concepción semántica de las teorías científicas”. Éndoxa: Series Filosóficas, UNED, Madrid, no. 8, pp. 41-91, 1997.

[21] Lorenzano, P., "La teorización filosófica sobre la ciencia en el siglo XX". Boletín de la Biblioteca del Congreso de la Nación, no. 121, pp. 29-43, 2001.

[22] Giere, R. No., La explicación de la ciencia. Un acercamiento cognoscitivo. México: Consejo Nacional de Ciencia y Tecnología. (Original en inglés de 1988), 1992.

[23] Izquierdo, M. y Adúriz-Bravo, A., "Epistemological foundations of school science". Science \& Education, vol. 12, no. 1, pp. 27-43, 2003.

[24] Develaki, M., "The model-based view of scientific theories and the structuring of school science programmes". Science \& Education, vol. 16, no. 7, pp. 725-749, 2007. DOI 10.1007/s11191-006-9058-2

[25] Koponen, I. T., "Models and modelling in physics education: A critical re-analysis of philosophical underpinnings and suggestions for revisions". Science \& Education, vol. 16, no. 7-8, pp. 751-773, 2007. DOI 10.1007/s11191-006-9000-7

[26] Amador Rodríguez, R. Y., Pujalte, A. y Adúriz-Bravo, A., Intervención y lenguajes: Dos tópicos epistemológicos que permiten indagar sobre la naturaleza de la ciencia. Memorias III Conferencia Latinoamericana de Historia, Filosofia y Didáctica de las Ciencias, Santiago de Chile. Chile. 17 y 19 de noviembre de 2014.

[27] Bliss, J., Ogborn, J. y Grize, F., The analysis of qualitative data. European Journal of Science Education, vol. 1, no. 4, pp. 427-440, 1979.

[28] Abd-el-Khalick, F., Waters, M. \& Le, A. P., "Representations of nature of science in high school chemistry textbooks over the past four decades". Journal of research in science teaching, vol., 45, no. 7, pp. 835-855, 2008. DOI 10.1002/tea.20226

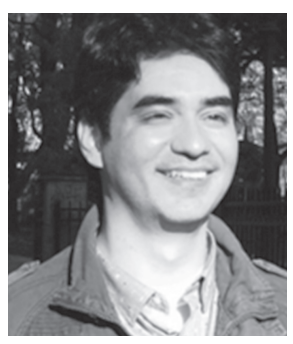

Rafael Amador-Rodríguez nació en Bogotá, Colombia, el 23 de marzo de 1976. Es Licenciado en Química y Magister en Docencia de la Química de la Universidad Pedagógica Nacional de Bogotá y Doctor en Enseñanza de las Ciencias Exactas y Naturales, mención Química de la Universidad Nacional del Comahue de Neuquén, Argentina. Áreas de Interés: Naturaleza de la ciencia; didáctica de la química e indagación escolar como competencia.

Natalia Ospina Quintero nació en Bogotá, Colombia, el 1 de mayo de 1986. Es Licenciada en Química de la Universidad Distrital Francisco José de Caldas y Doctoranda en Química Biológica de la Facultad de Ciencias Exactas y Naturales de la Universidad de Buenos Aires, Argentina. Áreas de interés: argumentación científica escolar; naturaleza de la ciencia. 


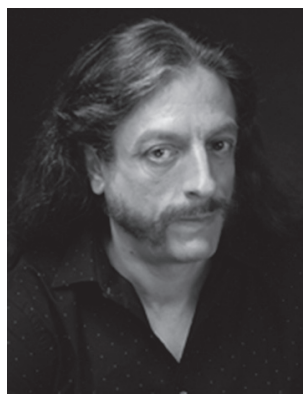

Agustín Adúriz-Bravo nació en Buenos Aires, Argentina, el 30 de agosto de 1971. Es Profesor de Enseñanza Media y Superior en Física por la Universidad de Buenos Aires y Doctor en Didáctica de las Ciencias Experimentales por la Universitat Autònoma de Barcelona. Áreas de interés: contribuciones de las metaciencias a la educación científica; formación epistemológica del profesorado de ciencias; naturaleza de la ciencia; modelización científica escolar; argumentación científica escolar. 